\title{
Estudio del uso que hacen los alumnos de los tests online voluntarios
}

\author{
Fidel Salas Vicente ${ }^{1}$ y Ángel Vicente Escuder ${ }^{1}$ \\ ${ }^{1}$ Departamento de Ingeniería Mecánica y de Materiales. Universitat Politècnica de València. \\ Camino de Vera, s/n. 46022. Valencia
}

\begin{abstract}
This paper presents an analysis of the use the students of a materials science course have done of a series of online tests that have been made available to them with the objective of providing them with a tool to practice before the evaluative exams and of giving them feedback about their learning process along the course.

The analyzed data show that altough the students have used extensively these tests and that has allowed them to obtain higher marks in the exams tests, they have done the tests only the days before the exams, answering rushedly and without obtaining a real benefit for their comprehension of the studied subject.
\end{abstract}

Keywords: online tests, time, learning

\section{Resumen}

En este trabajo se ha hecho un análisis del uso que hacen los alumnos de una asignatura de ciencia de materiales de una serie de tests en línea que se ha puesto a su disposición para que practiquen de cara a los exámenes parciales y obtengan información sobre su proceso de aprendizaje a lo largo de todo el curso.

Los datos recogidos muestran que si bien los alumnos hacen uso de estos tests con prodigariedad y les sirve para obtener una mejor nota el test del examen, los hacen solamente en los días previos a los exámenes, contestando de forma apresurada y sin que les sirva realmente para aumentar su comprensión de la materia estudiada.

Keywords: tests en línea, tiempo, aprendizaje 


\section{Introducción}

El uso de herramientas online para la docencia es ya una de las piedras angulares de los procesos educativos actuales, tanto para interactuar con los alumnos como para poner a su disposición todo el material que precisan para su formación, haya sido generado por el profesor o se haya tomado de los enormes recursos de acceso libre disponibles en la red.

Las herramientas informáticas para trabajo en línea no se adaptan aún muy bien a la realización de exámenes que consistan en pruebas escritas como la resolución de problemas en las que el alumno escribe fórmulas y las corrige a gran velocidad. Es cierto que un sistema de escritura digital podría suponer una mejora respecto al uso de lápiz y papel al evitar los tachones, cambiándolos por un limpio borrado del texto a descartar, pero la resolución que proporcionan estos dispositivos todavía no es la más adecuada ni su uso está extendido entre los alumnos. Lo contrario sucede con las preguntas tipo "respuesta múltiple" o similares, las cuales requieren solamente una mínima acción por parte del usuario y facilitan enormemente la corrección automática mediante métodos informáticos.

Esto ha llevado a que el uso de los tests en línea de respuesta rápida se haya extendido enormemente tanto para realizar exámenes evaluativos como para que los alumnos practiquen con ellos antes del examen. Esta última opción es muy demandada por los alumnos y parece llevar a un incremento de la nota final obtenida (Pennebaker, Gosling y Ferrell 2013; McDermott y col. 2014; Roediger y col. 2011; Cantor y col. 2014), aunque hay autores que dudan de la relación de causalidad, es decir, si lo que sucede es que los mejores alumnos hacen más tests o que hacer más tests les lleva a obtener mejores notas (Johnson 2006; Peat y Franklin 2003) o incluso, en algunos casos, de que la relación sea positiva (Zorio y Merello 2016). En cualquier caso, parece seguro que obtener una buena calificación en los tests preparatorios va acompañado de una buena nota en el test correspondiente a los exámenes evaluativos (Dobson 2008) y que los alumnos los encuentran beneficiosos, aunque solo sea porque les da mayor seguridad al haberlos enfrentado previamente a una prueba similar al examen (Rolfe y McPherson 1995) y les proporciona continuamente información sobre su nivel de aprendizaje (Rolfe y McPherson 1995; Iahad y col. 2004).

Finalmente, cabe destacar que el uso de tests en línea facilita la tarea del profesor y puede hacerlos más atractivos para lo alumnos actuales, acostumbrados al uso del móvil y el ordenador, pero en cuanto a la calificación final obtenida no supone un claro beneficio respecto a hacer los mismos tests con lápiz y papel (Anakwe 2008), además, es preciso que el alumno haga un buen uso de los tests para mejorar su comprensión de la materia y no solo para conseguir aprobar (Brothen y Wambach 2001).

En este trabajo se ha estudiado el uso que hacen los alumnos de los tests online que los profesores de una asignatura de ciencia de materiales han puesto a su disposición: número de alumnos que los hacen, cantidad de tests realizados a lo largo del curso y tiempo empleado en los tests, y se ha comparado con la nota final obtenida en la parte de test de los exámenes evaluativos de la asignatura. Finalmente se hacen unos comentarios sobre la influencia real que pueden estar teniendo estos tests sobre el proceso de aprendizaje del alumnado. 


\section{Objetivos}

El objetivo del presente trabajo ha sido obtener información sobre como los alumnos usan los tests en línea que tienen disponibles para cada tema de una asignatura de ciencia de materiales y cual es el efecto que la realización de estos tests durante el curso tiene sobre la calificación final obtenida en la parte de los exámenes evaluativos consistente en un test similar a los realizados en línea.

Para ello se han estudiado las siguientes variables:

- Cuando se han hecho los tests

- Cuantos tests se han hecho

- Cuanto tiempo se ha dedicado a los tests

- Calificaciones obtenidas en el test de los exámenes evaluativos

\section{Desarrollo de la innovación}

La asignatura de Materiales del Grado en Ingeniería en Diseño Industrial y Desarrollo de Productos, impartida en la Escuela Técnica Superior de Ingeniería del Diseño de la Universitat Politècnica de València, se divide en 14 unidades temáticas y, excepto para la primera unidad, que es de introducción, durante el curso 2019-2020 se ha dado a los alumnos la posibilidad de realizar una serie de tests en línea con el fin de que se puedan preparar para los exámenes evaluativos y puedan recibir información sobre su nivel de comprensión de la materia estudiada de forma continua.

Esta es la primera vez que se lleva a cabo esta acción, puesto que en años anteriores se realizaba un test online por cada tema, pero eran evaluativos y el alumno no tenía la oportunidad de practicar previamente más que mediante los medios que por su cuenta encontrase.

Los tests constan de 10 preguntas de respuesta múltiple, de completar, relacionar o similares en los que solamente una es respuesta es correcta y no hay límite de tiempo. Cada vez que se realiza un test las preguntas se escogen aleatoriamente de una base de datos preparada en años anteriores, de forma que algunas preguntas pueden repetirse en varios tests. La base de datos contiene entre 30 y algo más de 100 preguntas para cada uno de los temas de la asignatura, aunque los alumnos desconoces esta información. Al finalizar cada test el sistema informático da la alumno la nota obtenida junto con la respuesta correcta a cada pregunta.

Con el fin de estudiar el comportamiento de los alumnos se han exportado los datos que la plataforma educativa de la Politécnica de Valencia, PoliformaT, basada en SAKAI, guarda sobre estos tests formativos (hora de comienzo, IP, duración, nota,...). Esto ha permitido disponer de datos sobre todos los test excepto los del tema 8, que por alguna razón no se podían exportar y, dado que en el momento en que se estaba preparando este estudio el servicio informático de la UPV ya estaba dedicando todos sus recursos a la preparación del sistema para funcionar durante la cuarentena impuesta para tratar de contener la pandemia causada por el SARS-CoV-2, no se pudo solucionar el problema.

Los datos extraídos son los correspondientes a un total de 132 alumnos, que hicieron entre todos 20358 tests, eso sin contar los del tema 8. Estos datos se han analizado mediante el uso de macros en Excel y se les ha dado la representación gráfica que se ha creído más conveniente en cada caso.

(c) EY-NC-ND 2020, Universitat Politècnica de València Congreso IN-RED (2020) 


\section{Resultados}

\section{1 ¿Cuando se hacen los tests?}

Los distintos tests han sido accesibles a los alumnos desde el inicio de cada uno de los bloques en los que se ha dividido la asignatura (cada bloque abarca varios temas), lo que implica que los podían hacer incluso antes de que se diese el tema en clase. La idea es que los alumnos puedan ir haciéndolos al mismo tiempo que se van estudiando los temas para asentar los conocimientos, pero la Figura 1 indica que los alumnos han elegido en su mayoría seguir otra táctica y la mayor parte de los test se concentran, como era de esperar, en las 2 semanas previas a los exámenes.

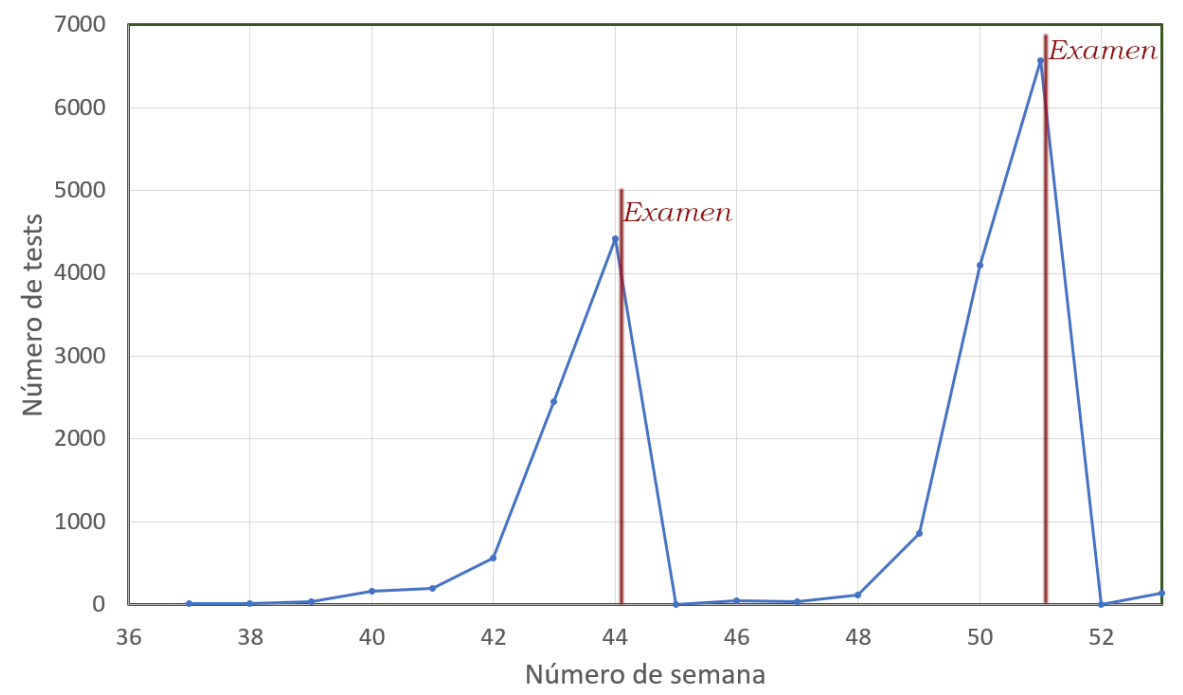

Fig. 1: Número de tests realizados por los alumnos en función del número de semana del año. Los números de semana superiores al 52 corresponden a Enero del año 2020.

Esto anula casi por completo el principal objetivo de los test, que es que el alumno use un sistema sencillo de autoevaluación formativa que le ayude a conocer sus deficiencias para corregirlas, pero a lo largo de todo el curso y no en los últimos días. Este comportamiento es una clara evidencia de que los objetivos del profesor -que el alumno aprenda- y del alumno son distintos, siendo el del alumno simplemente "aprobar". No quiere decir esto que no hayan alumnos con deseo de aprender, pero superar un examen, aún sin haber aprendido nada, es en general más importante para ellos que la mera adquisición de conocimientos.

\section{2 ¿Cuantos tests se hacen?}

Aunque los test se hacen de forma concentrada los días previos a los exámenes, la cantidad que se ha hecho es muy grande, con una media de 155 por alumno, lo que supone también unos 14 por alumno y tema. La media de tests por tema (véase la Figura 2) es de unos 1400 antes del primer parcial (hay 2 exámenes parciales en la asignatura), pero sube a más de 2300 tras ese primer parcial, posiblemente debido a que los alumnos han sentido la necesidad de mejorar sus calificaciones (la nota media en el test para el primer parcial fué de 7,6, bastante alta). Debe aquí recordarse que 
la inmensa mayoría se hicieron antes del examen y no cuando se impartieron las clases de teoría y prácticas de laboratorio correspondientes a cada tema.

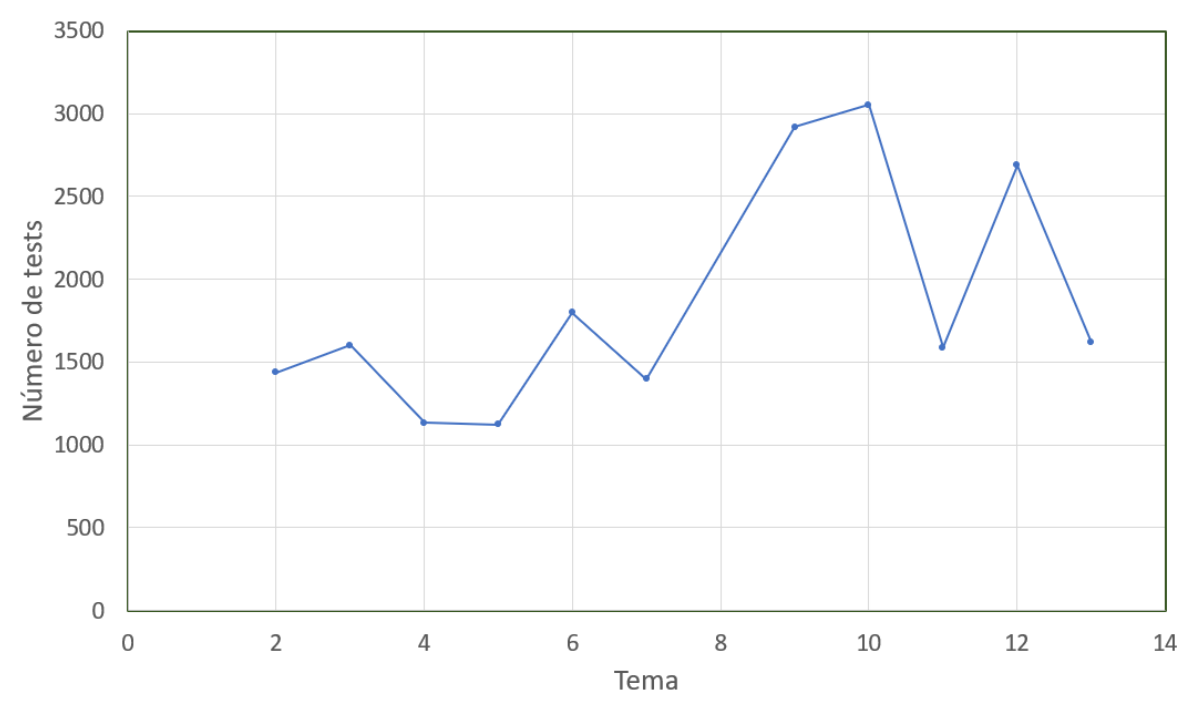

Fig. 2: Número de test realizados por los alumnos para cada tema del curso.

Si se estudia con más detalle cuantos tests hace cada alumno, se llega a la Figura 3. Esta figura muestra claramente como más de la mitad de los alumnos hizo menos de 10 tests por tema en el primer parcial, mientras que para el segundo parcial esa cantidad de alumnos se redujo notablemente, aumentando apreciablemente el porcentaje de los que hicieron más de 20 tests. Esto indica que si bien una parte de los alumnos siguió manteniendo la costumbre de no hacer uso de los tests -más del $20 \%$ no hizo ni 5 por tema e incluso aumentó la cantidad de los que hicieron 1 o ninguno-, la mayoría de ellos sí que aumentaron el uso de la herramienta.

\section{3 ¿Cuanto tiempo se dedica a hacer los tests?}

El estudio del tiempo que los alumnos dedican a resolver los tests es lo que más sorpresas ha deparado. Un rápido vistazo a la Figura 4 basta para comprender la razón.

Normalmente se acepta que 1 minuto por cada pregunta de tipo test es un valor aceptable en un examen (Brothen 2012), habiéndose visto incluso que dar un tiempo excesivo lleva a un descenso en las calificaciones obtenidas (Portolese, Krause y Bonner 2016; Salas y Vicente 2019). En este caso no todas las preguntas se basan en elegir una respuesta, pero el tiempo que se pueden requerir los otros tipos de preguntas se puede considerar similar Eso supone que el tiempo empleado para terminar los tests debería estar alrededor de 10 minutos. No obstante, la mayor parte de los alumno termina en 5 minutos o antes, exactamente el $65 \%$ de ellos. Además, el $30 \%$ de los test se terminaron en 2 minutos o menos y el $11 \%$ en 1 minuto o menos. Esto es algo que requiere ser estudiado con más detalle.

Se pueden plantear tres alternativas que expliquen estos tiempos tan bajos:

- Los datos recogidos por el sistema informático de la UPV son incorrectos 


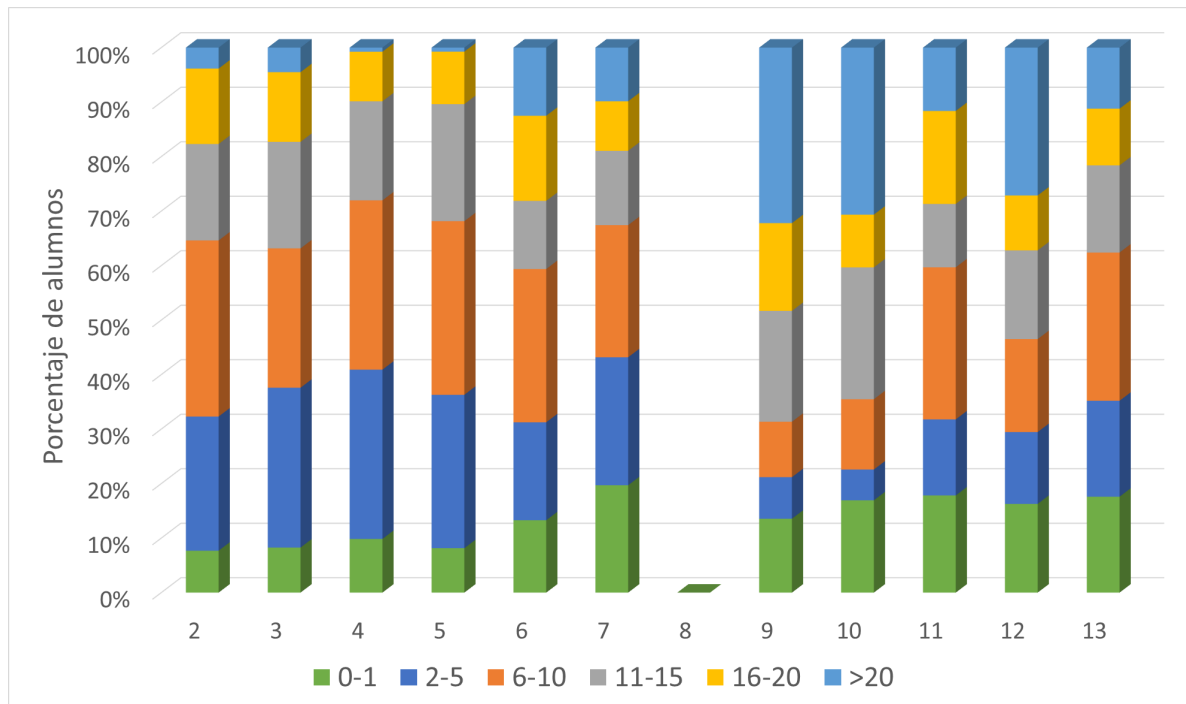

Fig. 3: Porcentaje de alumnos en función del número de tests que hicieron antes de los exámenes parciales y del tema estudiado.

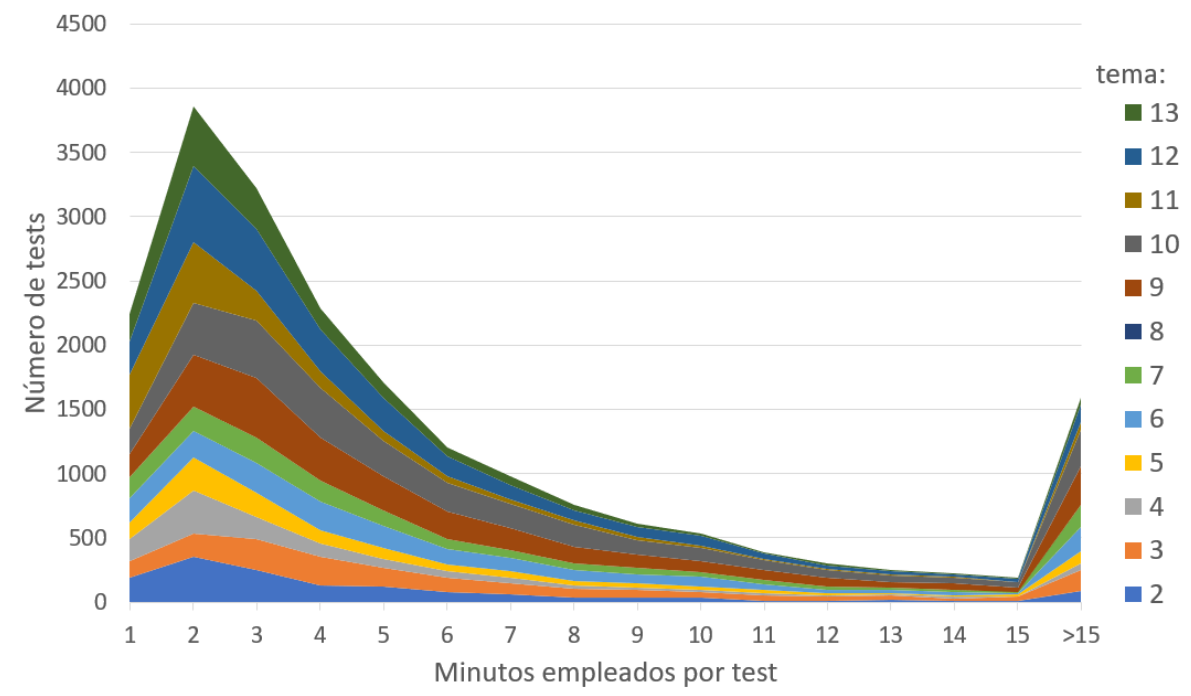

Fig. 4: Tiempo dedicado por los alumnos a la resolución de los tests. 
- Esos tiempos son reales pero están asociados a notas muy bajas o tests que se enviaron sin estar acabados.

- Esos tiempos son correctos y, por lo tanto, los test se han hecho de forma extremadamente rápida.

La primera opción es la que inicialmente puede considerarse más probable, pero un análisis de los tiempos recogidos para exámenes de otros años parece indicar que no hay razón para sospechar que los tiempos recogidos automáticamente sean falsos.

Es seguro que la segunda opción proporciona una parte de la explicación, y así lo muestra la Figura 5, con una gran cantidad de ceros, un $28.5 \%$, cuando el tiempo empleado es menos de 1 minuto. Parte de estos tests pueden explicarse porque el alumno ha interrumpido el trabajo y ha enviado el test sin contestar, pero la opción más plausible es que estuviese buscando ejemplos de preguntas nuevas y no se entretuviese en contastar las que ya conocía, por lo que al llegar a la última pregunta ha enviado el examen sin contestar para pasar a hacer otro test.

No obstante, con ese menos de un minuto empleado en el test también hay un $46.9 \%$ de notas igual a 10. Así pues, si bien la segunda opción es correcta, es preciso dar por buena también la tercera opción. ¿Como es esto posible?. Físicamente es posible contestar correctamente el test en menos de 1 minuto, pero solamente si se conoce la respuesta a las preguntas de antemano. Esto es exactamente lo que está sucediendo: la avidez de algunos alumnos por prepararse para el examen practicando con los test online es tan grande (hay algún alumno que ha hecho más de 150 tests para un tema) que les lleva a repetirlos una y otra vez. El problema es que las preguntas se repiten y tras unos cuantos ensayos ya conocen la respuesta a todas ellas, por lo que pueden contestar sin llegar a leer la pregunta, simplemente porque visualmente la reconocen.

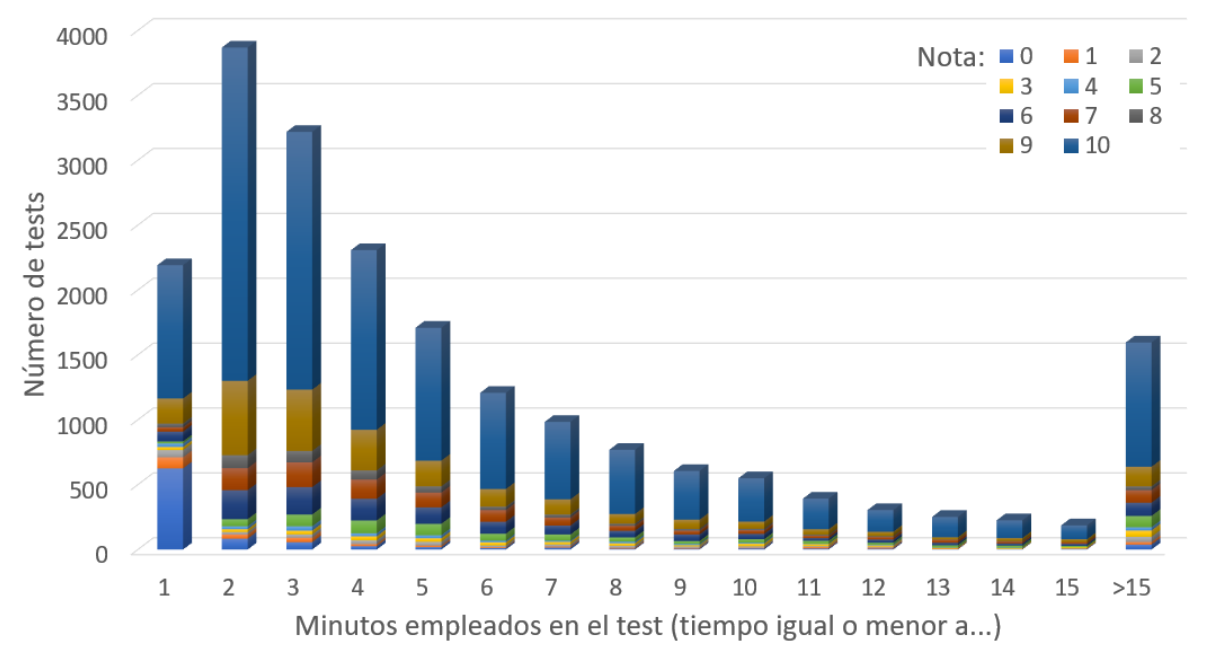

Fig. 5: Notas obtenidas en los tests en función del tiempo empleado en ellos.

Esto supone otro error de los alumnos, puesto que repetir de esta manera los test no les lleva a mejorar su aprendizaje e incluso puede darles una falsa sensación de seguridad al sentirse seguros contestando correctamente de forma mecánica unas preguntas que no han llegado a comprender. Esto es por lo tanto, algo a tener en cuenta por parte de los docentes. 
La Figura 6 muestra una información relacionada con lo que se está comentando: la nota media obtenida en los tests preparatorios en función del número de tests realizados. Esa figura muestra claramente que aunque el incremento en la nota que se va obteniendo al hacer más tests es inicialmente elevado, a partir de los 6 tests la mejora es muy escasa, aunque la nota media no pasa del 9. Esto sucede tanto para los temas en los que la cantidad de preguntas distintas en la base de datos es superior a 100 y en los que es bastante inferior, lo que denota cansancio por parte del alumno o que, como se ha comentado, ya conoce las respuestas.

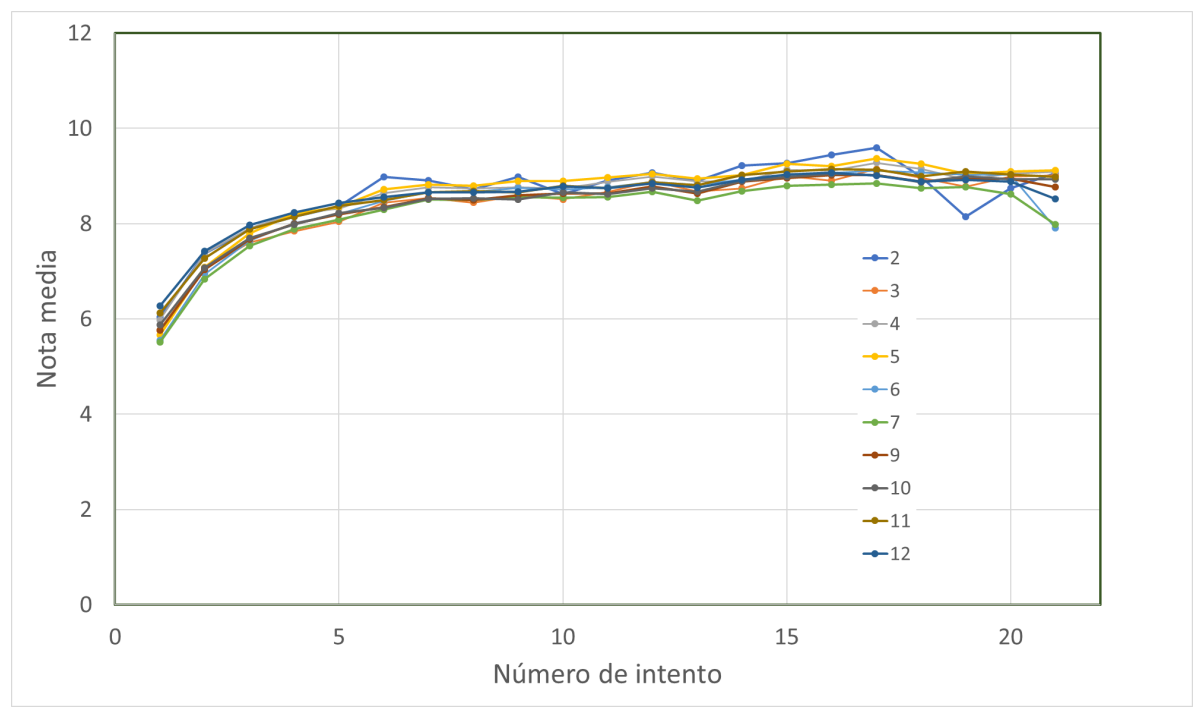

Fig. 6: Nota media obtenida en los tests en función del número de intentos. Cada línea representa un tema distinto.

\section{4 ¿Como influye la realización de tests en la nota final?}

Los tests evaluativos se realizaron al final de cada parcial de forma escrita y consistieron en preguntas similares a las incluidas en los tests formativos, aunque en ningún caso se usaron las preguntas de estós últimos.

Es de suponer que a mayor cantidad de tests realizados por el alumno, mayor será la nota que obtendrán en los exámenes evaluativos, por lo menos en la parte consistente en un test. Esta circunstancia se ve reflejada en la Figura 7.

La evolución de la nota media obtenida en función del número de tests hechos a lo largo del curso es muy similar en los dos parciales realizados, con un crecimiento casi contínuo que lleva a incrementar la nota media desde 5,97 a 9,2 en el primer parcial y desde 5,44 a 8,62 en el segundo parcial. En porcentaje, esto supone una mejora del 54 y el $58 \%$ respectivamente. No obstante, en ambos casos aparece un descenso inesperado de la nota para aquellos alumnos que han hecho algo más de 200 tests, unos 18 tests por tema. Encontrar una explicación a este fenómeno requerirá seguramente de un análisis más detallado del comportamiento seguido por los alumnos que se encuentran en ese intervalo en relación a los tests.

Queda por añadir que si bien las notas obtenidas en los tests de los exámenes parciales parecen mejorar notablemente gracias a los tests online, la nota media que obtuvieron los alumnos en la parte de problemas fue muy baja, de solo 3,26 sobre 10. Esto indica que si bien los alumnos han 


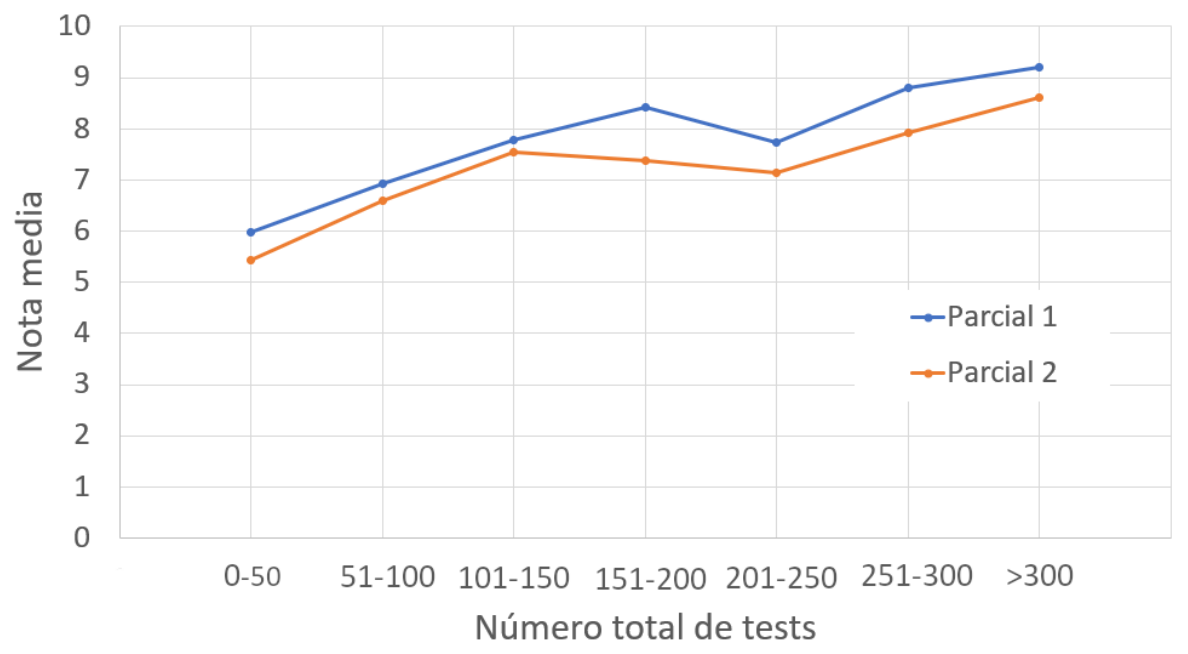

Fig. 7: Nota obtenida en la parte de tests de los exámenes evaluativos en función del número total de tests hechos por el alumno.

tenido una herramienta para mejorar su proceso educativo, han echo un uso equivocado de ella, centrándose en aprobar asegurando una buena nota en una parte del examen mientras dejan de lado la parte correspondiente a los problemas, parte que les requiere mayor esfuerzo mental, mayor integración de conocimientos y mayor comprensión de la materia.

\section{Conclusiones}

El análisis efectuado de los datos disponibles permite llegar a las siguientes conclusiones:

- Los alumnos encuentran atractivo y util disponer de test en línea para prepararse de cara al examen y la mayoría hace un uso extensivo de ellos.

- Los alumnos concentran la realización de los tests en los días previos a los exámenes parciales, perdiendose el objetivo principal de los tests, que es ayudar a la comprensión de la materiaria e informar al alumno sobre su nivel de aprendizaje a lo largo del curso.

- El tiempo empleado en completar los tests en muy bajo, lo que indica que muchas preguntas se contestan porque la respuesta se conoce de antemano y sin meditar sobre ella.

- A partir del sexto test realizado (por tema) la nota que se obtiene en los tests preparatorios deja de aumentar.

- Pese a ello, los datos indican que cuantos más tests se hacen, aunque las preguntas sean repetidas, mayor es la nota que se obtiene en los tests de los exámenes evaluativos.

Es preciso destacar que los resultados obtenidos hacen referencia a la nota obtenida en los tests de los exámenes y no a la nota total obtenida en la asignatura, la cual incluye también una parte de resolución de problemas (cuya nota media es muy baja) y las prácticas de laboratorio. Es por ello que los resultados presentados no son válidos para asegurar que los tests online han ayudado a 
mejorar la comprensión real de la materia estudiada, puesto que el comportamiento de los alumnos muestra estar enfocado a aprobar el examen de una forma mecanicista.

\section{Referencias bibliográficas}

Anakwe, Bridget (2008). "Comparison of Student Performance in Paper-Based Versus ComputerBased Testing". En: Journal of Education for Business 84.1, págs. 13-17. DOI: 10.3200/joeb.84. 1.13-17.

Brothen, T. y C. Wambach (2001). "Effective Student Use of Computerized Quizzes". En: Teaching of Psychology 28.4, pág. 292. ISSN: 0098-6283.

Brothen, Thomas (2012). "Time Limits on Tests". En: Teaching of Psychology 39.4, págs. 288-292. DOI: $10.1177 / 0098628312456630$.

Cantor, Allison D. y col. (2014). "Multiple-choice tests stabilize access to marginal knowledge". En: Memory \& Cognition 43.2, págs. 193-205. DOI: 10.3758/s13421-014-0462-6.

Dobson, John L. (2008). "The use of formative online quizzes to enhance class preparation and scores on summative exams". En: Advances in Physiology Education 32.4, págs. 297-302. DOI: 10.1152/advan.90162.2008.

Iahad, N. y col. (2004). "Evaluation of online assessment: the role of feedback in learner-centered elearning". En: 37th Annual Hawaii International Conference on System Sciences, 2004. Proceedings of the. IEEE. DOI: 10.1109/hicss.2004.1265051.

Johnson, G. (2006). "Optional online quizzes: College student use and relationship to achievement". En: Canadian Journal of Learning and Technology 32.1. ISSN: 1499-6677.

McDermott, Kathleen B. y col. (2014). "Both multiple-choice and short-answer quizzes enhance later exam performance in middle and high school classes." En: Journal of Experimental Psychology: Applied 20.1, págs. 3-21. DOI: 10.1037/xap0000004.

Peat, Mary y Sue Franklin (2003). "Has student learning been improved by the use of online and offline formative assessment opportunities?" En: Australasian Journal of Educational Technology 19.1. DOI: 10.14742 /ajet.1703.

Pennebaker, James W., Samuel D. Gosling y Jason D. Ferrell (2013). "Daily Online Testing in Large Classes: Boosting College Performance while Reducing Achievement Gaps". En: PLoS ONE 8.11. Ed. por publisher = Public Library of Science (PLoS) Manuel João Costa, e79774. DoI: 10.1371/journal pone. 0079774.

Portolese, Laura, Jackie Krause y Julie Bonner (2016). "Timed Online Tests: Do Students Perform Better With More Time?" En: American Journal of Distance Education 30.4, págs. 264-271. DOI: 10.1080/08923647.2016.1234301. 
Roediger, Henry L. y col. (2011). "Test-enhanced learning in the classroom: Long-term improvements from quizzing". En: Journal of Experimental Psychology: Applied 17.4, págs. 382-395. DOI: $10.1037 / \mathrm{a} 0026252$.

Rolfe, I. y J. McPherson (1995). "Formative assessment: how am I doing?" En: The Lancet 345.8953, págs. 837-839. DOI: 10.1016/s0140-6736 (95)92968-1.

Salas, F. y A. Vicente (2019). "Influencia del tiempo disponible y usado en un examen online sobre la nota obtenida". En: 27 Congreso Universitario de Innovación Educativa en las Enseñanzas Técnicas. Alcoi, págs. 381 -389.

Zorio, A. y P. Merello (2016). "Efectos en el examen final de la participación del estudiante en cuestionarios online". En: Libro de Actas IN-RED 2016 - II Congreso Nacional de Innovación Educativa y de Docencia en Red. DOI: 10.4995/inred2016.2016.4345. 